\title{
Convergencias y divergencias en la frontera norte de México
}

\author{
Mario M. Carrillo Huerta \\ El Colegio de Puebla
}

\section{Resumen}

El presente estudio aborda de qué manera lo ocurrido en la frontera México-Estados Unidos afecta la vida total de los dos países, ya que en esta región se presentan procesos de interacción socioeconómica y cultural que involucran componentes nacionales de ambas naciones. Para explorar este proceso de latinoamericanización, se desarrolló un modelo para calificar los efectos del intercambio internacional que tiene lugar entre los dos países. En dicho modelo el cambio cultural y la adaptación económica son en función de la intensidad de los flujos de intercambio, y de la receptividad o disponibilidad/habilidad al cambio del país anfitrión. Se concluye que la región fronteriza méxico-americana es un punto donde convergen el proceso de latino-americanización de Estados Unidos y la americanización de Latinoamérica, procesos concomitantes; aunque heterogéneos y asimétricos.

Palabras clave: intercambio cultural, americanización, latinoamericanización, interdependencia, inversión extranjera.

\begin{abstract}
This essay explains how the events that take place in the border between Mexico and the United States affect the whole life of the two countries, where processes of socio-economical and cultural interaction involving the proper components of the two countries appear. To explore this Latin-Americanization process, it was developed a model to measure the effects of the international exchange between the two countries. Cultural changes and economic adaptability of these models are conditioned by the intensity of exchange flow and the receptivity or capability of the host country to adjust to these changes. The conclusion is that the border Mexican-American region is a convergent point to the Latin-Americanization of the United States as well as the Americanization of Latin America, the concomitant, but heterogeneous and asymmetric processes.
\end{abstract}

Keywords: cultural exchange, Americanization, Latin-Americanization, interdependence, foreign investment. 


\title{
CONVERGENCIAS Y DIVERGENCIAS EN LA FRONTERA NORTE DE MEXICO*
}

\author{
Por \\ Mario M. Carrillo Huerta \\ El Colegio de Puebla, A.C.
}

\section{INTRODUCCION}

El presente trabajo trata sobre la forma en que lo que sucede en la frontera de México con Estados Unidos afecta la vida de los dos países en general. Aquí se define a la frontera como una región formada por las áreas geográficas de ambos países contiguas a lo largo de la línea divisoria internacional y en las que se suceden procesos de interacción socioeconómica y cultural que involucran componentes nacionales de ambos países.

De acuerdo con esa perspectiva conceptual, la región fronteriza MéxicoEstados Unidos es un punto donde convergen dos procesos concomitantes aunque heterogéneos y asimétricos: la latinoamericanización de Estados Unidos y americanización de Latinoamérica.

El trabajo se ha dividido en tres secciones. En la primera se presenta un marco de referencia para evaluar la forma en que los flujos internacionales de bienes, servicios, personas y tecnología pueden conducir a la asimilación económica y cultural. En la segunda, se examinan esos flujos para el caso de la región fronteriza, mientras que en la tercera se presentan las conclusiones del trabajo.

\section{INTERCAMBIO INTERNACIONAL Y COMPORTAMIENTO CULTURAL Y SOCIOECONOMICO}

\section{Introducción}

El intercambio entre dos países puede afectar el comportamiento cultural y socioeconómico, en la medida en que los artículos intercambiados lleven con ellos características culturales y/o económicas incorporadas. Claramente, los efectos culturales y socioeconómicos de los flujos de intercambio internacional en el país anfitrión dependerán de la naturaleza e intensidad de los flujos y de la receptividad o disposición de cambiar del país an-

\footnotetext{
* Texto originalmente escrito en el idioma inglés y presentado en el Seminario sobre "The Latinamericanization of the United States" celebrado en Tucson, Arizona en abril 2-5 de 1982. En este trabajo, la frontera México-Estados Unidos será considerada como la compuesta por los estados fronterizos de aquellos países. Sólo cuando se especifique, la frontera se definirá de otra manera.
} 
fitrión: mientras más intensos son los flujos y más alto es el grado de tal receptividad, más fuertes serán los efectos esperados y más fuerte la reacción del país anfitrión en la forma de cambio cultural y adaptación socioeconómica. ${ }^{1}$

Con respecto a la naturaleza de los flujos de intercambio, uno puede esperar un contenido más alto de características culturales y/o socioeconómicas en artículos tecnológicos que en los no tecnológicos. Siendo definida la tecnología como el proceso de interacción entre el hombre y las herramientas, sugiere que los flujos de gente, herramientas e información llevan más características culturales que cualquier otro tipo de flujo. ${ }^{2}$ Esto es como debiera ser, pues la gente define y es el principal aspecto de la cultura. ${ }^{3}$

Considerando la clasificación económica tradicional de artículos intercambiados, se podría entonces decir que los factores de producción, conllevan más aspectos culturales que los productos finales y servicios, y que, entre otros factores, el trabajo y la empresarialidad son los más importantes portadores, seguidos por el capital en su forma física. ${ }^{4}$ Otro importante portador cultural que no podría ser específicamente económico en naturaleza es la información.

Efectivamente, la información comúnmente acompaña los flujos tanto de los factores de producción como de los productos finales y servicios, pero puede ser un portador cultural en sí sin estar relacionado con tales flujos. Este es el caso, por ejemplo, de la información que fluye al través de medios masivos de comunicación (radio, TV y otros).

1 Esta sería la visión institucionalista-instrumentalista, la cual supone que el comportamiento humano es determinado por la combinación de impulsos biológicos y acondicionamientos sociales. Según esta visión, el intercambio internacional ensancharía los acondicionamientos sociales. Ver, por ejemplo: John Dewey, Human nature and conduct (New York: The Modern Library, 1957)

2 Esta es la definición institucionalista de tecnología. Los institucionalistas ven el comportamiento humano como dicotómico, con manifestaciones tecnológicas y ceremoniales. Las tecnológicas son las acciones en las cuales los humanos usan herramientas, mientras que las ceremoniales son las acciones en las que las herramientas son usadas para la creación y apoyo de mores y tabúes. Vér, por ejemplo: Clarence Ayres, The theory of economic progress (New York: Schocken Books, 1944).

3 Si la cultura es "ese todo complejo que incluye conocimiento, creencia, arte, moral, ley, costumbre y cualesquiera otras capacidades y hábitos adquiridos por el hombre como miembro de una sociedad", entonces el hombre es el poseedor de cultura más importante. Véase: Leslie A. White, The science of culture: A study of man and civilization (New York: Grove Press, Inc., 1949)

4 Capital físico es definido como el conjunto de bienes producidos los cuales entran, directa o indirectamente, dentro de la producción de todos los bienes. Incluye instrumentos, maquinaria, planta y equipo. Este tipo de capital es diferente del financiero yue ". . . es propiedad de los medios de producción que permiten a los propietarios de iguales cantidades de ganar iguales beneficos (bajo condiciones de competencia)". Véase Edward J. Nell "Theories of Growth and Theories of Value", Economic development and cultural change XVI (1967), pp. 15-26 
La intensidad de los flujos de intercambio depende de su tamaño y duración. Mientras más grande sea el tamaño y más permanente sea, la intensidad del flujo será mayor.

En términos de la receptividad, puede decirse que ésta depende principalmente del carácter, la estructura y la etapa de desarrollo de la sociedad. Una sociedad abierta se espera que sea más receptiva a las importaciones culturales que una cerrada. También, la diversificación étnica y económica incrementa las posibilidades de una mayor receptividad a la importacion cultural. Por otro lado, la aprerturá y diversificación de la sociedad se incrementan con el desarrollo.

Lo anterior sugiere que mientras más intenso sea el intercambio de portadores de caracteres culturales y socioeconómicos entre las sociedades y más receptivas sean esas sociedades, mayor será el grado de cambio cultural y adaptación económica de cada una de ellas hacia la naturaleza y estructura de la otra; esto es, mayor será el grado de convergencia.

El resto de esta sección se refiere a los tipos de flujos, efectos y resultados, que pueden ser esperados del intercambio entre México y los Estados Unidos en general, y entre su frontera común en particular.

\section{Intercambio internacional entre México y Estados Unidos}

Características generales. México y Estados Unidos, participan en un intenso intercambio de bienes, servicios e información. Este intercambio es tan intenso, que alrededor del 60 por ciento del total de comercio externo de México es con Estados Unidos, mientras que México es el tercer socio comercial más importante de Estados Unidos. ${ }^{5}$

Debido principalmente a sus respectivas etapas en el proceso de desarrollo, lo cual se refleja en su estructura socioeconómica al través de su diferente abundancia relativa de factores de producción, la naturaleza del intercambio entre los dos países es tal que Estados Unidos exporta capital (tanto físico como financiero), productos terminados intensivos en capital y empresarialidad, mientras que importa trabajo, productos intensivos en trabajo y materias primas de México. ${ }^{6}$ Por otra parte, por el hecho de ser

5 En efecto, en 1980, el comercio de México con EUA ascendió a alrededor de 67 de su comercio exterior total. También en 1980, México fue el tercer socio más importante de EUA, después de Canadá y Japón. Ese año, el valor de las exportaciones a Canadá ascendieron a 16 de las exportaciones totales de EUA; las exportaciones a Japón ascendieron a 9.4 del total, mientras las exportaciones a México ascendieron a 6.9 del total. Véase: U.S. Departmento of Commerce, Statistical abstracto of the United States, 1981.

6 Según Reynolds, “ . . . Los Estados Unidos han representado tradicionalmente más de 70 del total de la Inversión Extranjera Directa en México". Véase: Clark W. Reynolds, "Labor Market Proyections for the United States and México and their Relevance to Current Migration Controversies", en Food Research Institute Studies, Vol. 17 
vecinos, una proporción considerable de su comercio se realiza al través del turismo que fluye en ambas direcciones, y un considerable intercambio de información se lleva a cabo al través de la televisión, la radio y otros medios de comunicación.

Pero el ser vecinos también permite que los flujos de bienes, servicios e información sean contínuos, de manera que al menos se asegura cierto grado de intensidad. Los otros elementos que califican la intensidad de los flujos: su tamaño y carácter general, pueden ser empíricamente contrastados.

Migración. El fenómeno migratorio es un ejemplo obvio de la interacción económica, social y cultural que tiene lugar entre México y los Estados Unidos, tanto a nivel nacional como a nivel regional en la frontera. Durante más de cien años, la migración ha sido el resultado de un verdadero fenómeno bilateral de interacción de elementos localizados en los dos lados de la frontera: una demanda de mano de obra barata en el lado de Estados Unidos y una oferta de fuerza de trabajo poco costosa en el lado mexicano. ${ }^{7}$ Esto no quiere decir que la migración mexicana a los Estados Unidos sea un fenómeno producido mecanicamente por la oferta y la demanda en los mercados de trabajo. Más bien, se sugiere aquí que este fenómeno no existiría sin esa interacción de elementos.

Mientras algunos autores han sostenido que la migración de trabajadores a los Estados Unidos ha sido permanente, ${ }^{8}$ estudios recientes de la migración de trabajadores mexicanos a ese país industrializado, han mostrado que una proporción considerable de dichos trabajadores, permanecen en los Estados Unidos por un período de alrededor de seis meses en promedio, y por lo tanto puede ser considerada como temporal. ${ }^{9}$ Permanente o temporal, el hecho es que siendo un flujo continuo a lo largo de cien años, la migración de trabajadores mexicanos a los Estados Unidos es el factor principal responsable de la proporción relativamente alta de mexicanos y/o gente de origen mexicano en la población total de ese país, la cual ha sido estimada en casi $4 \% .10$

7 Véase por ejemplo, Michael J. Piore, Birds of passage, Migrant labor and industrial societies)Cambridge Mass.: University Press, 1979), pp. 51-55.

8 Ibid., pp. 50-60. Esto se refiere a la migración que se origina en México como un todo. Otro tipo de migración de trabajadores que se origina y termina en la frontera, será analizada en la siguiente sección.

9 César Zazueta, "Investigación reciente sobre migración mexicana indocumentada a los Estados Unidos", trabajo presentado en el Seminario Latinoamericano en la Universidad de Harvard, Febrero 19, 1980. p. 78 .

10 De hecho en 1980, cerca del 6.4 del total de la población de Estados Unidos eran personas de origen hispánico, y 60 de ellas eran de origen mexicano. Ver: U.S. Bureau of the Census, 1980 Census of Population, Supplementary Report, PC 80-S1-1, y Current Population Reports Series p-20, No. 361 . 
Por otro lado, el flujo de inmigrantes mexicanos a los Estados Unidos ha sido tal vez el más importante en volumen en tiempos recientes. A causa de ésto y de su carácter continuo, uno puede afirmar que el flujo de trabajadores mexicanos es comúnmente el de mayor influencia en ambos aspectos, cultural y económico.

Otro elemento importante que la migración agrega al de intercambio cultural es su efecto positivo en la estructura étrica en la sociedad norteamericana que a su vez incrementa la posibilidad de cambio cultural y/o adaptación económica al través de un mayor grado de receptividad cultural. Las importaciones culturales mexicanas, refuerzan los elementos también culturales de los grupos méxico-norteamericanos, además de su impacto sobre el resto de la población norteamericana.

De cualquier manera, las características anteriormente citadas del principal flujo de intercambio que va de México a Estados Unidos, son definitivamente diferentes a las del principal flujo de Estados Unidos a México.

Movimiento de capital. En cambio a los factores de la producción, el principal intercambio que fluye de los Estados Unidos a México incluye capital físico y financiero al través de la inversión extranjera. Como se ha mencionado ya, en términos de sus efectos sobre la cultura del país receptor, es claro que el capital físico (equipo, maquinaria y herramientas) es el factor de mayor influencia ya que lleva consigo información tecnológica. ${ }^{11}$

Una versión importante de los flujos de capital físico, son las plantas ensambladoras o maquiladoras que están localizadas en México y producen para el mercado norteamericano con capital, equipo y materia prima norteamericana. ${ }^{12}$ Como un apéndice a la ley industrial mexicana, el programa de maquiladoras comenzó a mediados de los años sesentas. Para 1982, México alojaba cerca de 600 maquiladoras.

El programa de maquiladoras ha sido probablemente el programa industrial individual más discutido en México desde que comenzó en 1965.

\footnotetext{
11 Por ejemplo, entre 1975 y 1978 , el valor de los bienes de capital representó un promedio de alrededor de 30 del valor total de las importaciones mexicanas. Véase: Banco de México, Balanza de Pagos 1970-1978 (México, D.F.: Banco de México, S.A., 1980).

12 El Programa de Industrialización Fronteriza empezó en 1965. En junio de 1966 se anunció la primera reglamentación general que autorizaba las importaciones temporales (libres de impuestos) de maquinaria, equipo y materiales para aquellas firmas ensambladoras que se localizarán en territorio mexicano a 20 kilómetros de la frontera con Estados Unidos. Tal reglamentación se extendió en 1971 para incluir las costas mexicanas en la zona permitida de instalación y fue posteriormente expandida para incluir todo el territorio mexicano. La legislación reguladora de maquiladoras más reciente se anunció en 1977 y continuaba permitiendo la importación libre de impuestos del capital físico y de materiales si las firmas producían enteramente para el exterior.
} 
Muchos estudios de maquiladoras tratan de evaluar el programa mediante la presentación y análisis de las ventajas y desventajas que representan para México, mientras que otros se relacionan con las características generales de operación de las plantas. Ha habido sólo un intento de evaluación del programa sobre la base de análisis de costo-beneficio. ${ }^{13}$

Ahora bien, no existe consenso en cuanto a si el programa de maquiladoras cuesta más de lo que beneficia al país o no. El hecho es que el programa ha sido contínuo y creciente desde su inicio. Esto, combinado con su relativamente alta importancia en términos de tamaño, hace del flujo de capital físico Estados Unidos-México, el más intenso y de mayor influencia económica.

Los otros flujos importantes se dan en ambas direcciones aunque con diferentes intensidades. Este es el caso del turismo y la información.

Turismo e información. La principal diferencia entre comercio y turismo es que este último agrega información cultural al anterior. De este modo, aunque menor en tamaño que el comercio, el turismo es más importante como portador cultural.

Entre México y los Estados Unidos, el turismo fluye en ambas direcciones, pero, ya que depende profundamente del ingreso de los turistas, este flujo es más importante en la dirección Estados Unidos-México que viceversa. 14 Más aún, el tipo de influencia del turismo en el país anfitrión es similar al de la migración, aunque es definitivamente menos permanente que el de cualquier tipo de migración.

La información a través de los medios de comunicación entre México y Estados Unidos, también fluye en ambas direcciones. Pero, debido a que el desarrollo de los medios de comunicación y sistemas de información depende de la etapa del desarrollo global de la sociedad, el flujo es de esperar que sea más importante en la dirección Estados Unidos-México que viceversa.

\footnotetext{
13 Wolfang Köning, "Efectos de la actividad maquiladora fronteriza en la sociedad mexicana, en Roque González Salazar, comp., La frontera del norte: integración y desarrollo (México, D.F.: El Colegio de México, 1981), pp. 95-105.
}

14 Por ejemplo, en 1978, alrededor del 82 del número total de turistas que visitaron México eran residentes de E.U. Este año, los gastos de los turistas norteamericanos ascendieron a alrededor del 80 del valor total de los gastos. Ese año casi 92 de turistas mexicanos, visitaron Estados Unidos y representaron casi el 72 del valor total de gastos de turistas mexicanos en el extranjero. Sin embargo, los gastos de turistas norteamericanos en México (806.6 millones de dólares) representaron alrededor de 218 con respecto a los gastos de turistas mexicanos en los E.U. (370.6 millones). Véase: Banco de México Balanza de Pagos 1970-1978 (México, D.F.: Banco de México, 1981), pp. 302-303-309 y 310 . 
Conclusiones de la sección. La principal conclusión que puede ser obtenida de esta sección es que debe haber convergencias culturales y socioeconómicas entre México y los Estados Unidos en general. La principal hipótesis, sin embargo, aún es que esas convergencias son más fuertes en las zonas fronterizas que a nivel nacional.

La siguiente sección incluye un análisis de la evidencia empírica de la distribución regional del principal flujo de intercambio entre los dos países, considerando sólo dos regiones: la frontera y el resto de los respectivos países.

\section{EVIDENCIA EMPIRICA DE LAS CONVERGENCIAS EN LAS ZONAS FRONTERIZAS MEXICO-ESTADOS UNIDOS.}

\section{Introducción}

La frontera México-Estados Unidos ha recibido recientemente una atención considerable por los estudios tanto mexicanos como norteamericanos. Indudablemente la razón de esto es el reconocimiento general de la frontera como el escenario de importantes procesos de interacción e interdependencia que son el resultado de un desarrollo paralelo de poblaciones en ambos lados. Siendo el lugar donde dos países se encuentran, una frontera debiera ser el escenario donde los fenómenos nacionales tienen sus más inmediatos y más aparentes efectos internacionales.

Tal vez el mejor ejemplo de cómo la frontera es impactada por los fenómenos nacionales, sea dado por la devaluación del peso mexicano en 1976-1982.15 Después de la devaluación, un número considerable de comercios en el lado norteamericano tuvieron que cerrar. La razón fue que el grueso de la demanda para su productos venía del otro lado de la frontera, y la devaluación hizo a todos los productos relativamente más caros para los mexicanos.

Además, debido a que muchos de los comercios en Estados Unidos empleaban trabajadores mexicanos que viajaban diariamente desde su lugar de residencia en México, los cierres causaron el despido de trabajadores mexicanos. A su vez, ésto ocasionó un decremento en los ingresos de la fuerza de trabajo mexicana del área. El resultado fue por consiguiente, una inmediata depresión en la economía de ambos lados de la frontera.

15 Para una detallada consideración de los efectos de la devaluación del peso de 1976, Véase: Ricardo Torres Gaytán, Un siglo de devaluaciones del peso mexicano (México: Siglo XXI Editores, 1980) pp. 352-353; 
La característica principal del proceso de interacción económica en la frontera es su asimetría. Esto es así porque tiene lugar entre dos economías que son diferentes no sólo en términos de tamaño sino también en términos de su estructura. Por una parte, ha sido estimado que alrededor del $40 \%$ más importantes tienen más del 30 por ciento de sus trabajadores empleados mía de San Diego. Sin embargo, las actividades económicas de Tijuana, representan alrededor del 5 por ciento derla economía de San Diego. ${ }^{16}$ Por otra parte, la economía de la frontera mexicana tiende a especializarse en actividades de servicio en mucho mayor grado que la economía de la frontera norteamericana. Mientras que la totalidad de los municipios mexicanos más importantes tienen más del 30 por ciento de sus trabajadores empleados en el sector de servicios, todas las áreas fronterizas de los estados norteamericanos de la frontera tienen menos del $30 \%$ de su empleo en servicios. ${ }^{17}$

Dentro del contexto del esquema analítico desarrollado en la sección previa, la frontera debiera sumar más puntos que el resto de los respectivos países en términos de la intensidad del flujo de intercambio entre México y Estados Unidos, pero debiera observar la misma pauta en la dirección de los principales flujos internacionales: trabajo, capital físico, turismo e información.

\section{Migración}

Los migrantes mexicanos, legales o indocumentados, a los Estados Unidos, han tenido de manera tradicional los estados del suroeste como su destino final. 18

En un estudio reciente de migración general de indocumentados, Robinson ha estimado que los estados del suroeste (Arizona, California, Colorado, Nuevo México y Texas) han recibido a más del $60 \%$ del total de los inmigrantes indocumentados a los Estados Unidos. 19 Más recientemente,

16 Ver: Norries C. Clement y Kenneth Shellhammer, "Estados Unidos-México Border Area Economic Interdependence: The Case of San Diego California, and Tijuana, Baja California." Trabajo presentado en la Primera Reunión de Impactos Regionales de las Relaciones México-Estados Unidos en Guanajuato, México, en Julio, 1981.

17 Para los datos de México, ver: Secretaría de Industria y Comercio, IX Censo General de Población 1970. Para los datos en los Estados Unidos, véase: Niles Hansen, The border economy. Regional development in the Southwest, (Austin, Tex.: University of Texas Press, 1981), p.56.

18 Manuel Gamio, Mexican Inmigration to the United States (New York: Dover Publications, 1971), y Número, procedencia y distribución geográfica de los inmigrantes mexicanos a Estados Unidos, (México, D.F.: Talleres Gráficos de la Nación; 1930).

19 J. Gregory Robinson, "Estimating the Approximate Size of the Illegal Alien Population in the United States by the Comparative - Trend - Analysis of Age - Specific Death Rates", en Demography Vo. XVII, No. 2 (1980), pp. 159-176. 
la investigación realizada por CENIET acerca de la migración de mexicanos indocumentados a los Estados Unidos ha mostrado que el destino más común de migrantes mexicanos a ese país continúa siendo el de los estados del suroeste, además del estado de Illinois. 20

Es importante hacer notar, sin embargo, que buena parte de los trabajadores mexicanos migrantes a la zona fronteriza terminan en el lado mexicano, ya sea permanente o temporalmente. ${ }^{21}$

Es también importante hacer notar que la migración a la frontera México-Estados Unidos no está limitada a mexicanos. Aunque todavía poco conocida, existe también migración originada en Centroamérica que cruza México en ruta hacia los Estados Unidos. ${ }^{22}$

En todo caso, tales migraciones pueden ser consideradas como de larga distancia con respecto a la frontera. Existe aún otro flujo de migración que se origina y termina en la frontera. Tal migración está compuesta de trabajadores mexicanos que van y vienen a las zonas fronterizas de Estados Unidos con la debida documentación, los que son conocidos como "transfronterizos". ${ }^{23}$ La mayor parte de esta migración, la cual está compuesta de trabajadores tanto mexicanos como norteaméricanos está en relación con todo tipo de actividades con una ligera tendencia hacia los servicios. La última estimación de tal flujo lo coloca con un volumen de 78,000 trabajadores. 24

\section{Capital físico: maquiladoras}

Con respecto al flujo de capital físico de los Estados Unidos a México, su distribución geográfica se está concentrando en la región fronteriza. El cuadro I muestra la distribución regional de las maquiladoras en México

20 César Zazueta, op. cit., p. 87.

21 La migración temporal a la frontera no se registra en las oficinas del censo ni en E.U. ni en México. Pero la presencia de ambas, la temporal y la permanente, se muestra por la alta tasa de crecimiento de la población experimentada, al menos en el lado mexicano de la frontera. Entre 1960 y $1970,1 a$ población total de los estados de la frontera mexicana creció a una tasa promedio de 3.54 por año, mientras que la tasa anual era del 3.28 para el país como un todo en ese período. Véase: Secretaria de Industria y Comercio, VIII y IX Censos Generales de Población y vivienda, 1960, 1970.

22 Esta no es necesariamente una inmigración en una sola dirección. Existen centroamericanos que en número creciente terminan su viaje en la frontera México-Estados Unidos, ya sea en camino a Estados Unidos o bien porque son forzados a regresar. Este fenómeno ha internacionalizado la frontera de su tradicional contexto bilateral, pero sigue permaneciendo sin registrarse.

23 Véase: Frida Espinosa Jaramillo, Condiciones de trabajo y situación socioeconómica del trabajador agricola transmigrante del Valle Imperial, Cuadernos de Ciencias Sociales, Serie 1-No 5, Instituto de Investigaciones Sociales de la Universidad Autónoma de Baja California.

24 Ibid., p. 18. La estimación es para 1975. 
CUADRO I. Empleo y valor agregado en plantas maquiladoras en México, por región: 1976-1981.

\begin{tabular}{|c|c|c|c|c|c|c|}
\hline \multirow[b]{2}{*}{ CONCEPTO } & \multicolumn{6}{|c|}{ A Ñ OS } \\
\hline & 1976 & 1977 & 1978 & 1979 & 1980 & 1981 \\
\hline \multicolumn{7}{|c|}{ 1. MAQUILADORAS } \\
\hline \multicolumn{7}{|l|}{ 1) TOTAL: } \\
\hline a) Número & 448 & 443 & 457 & 540 & 620 & 616 \\
\hline b) Porcentaje & 100.0 & 100.0 & 100.0 & 100.0 & 100.0 & 100.0 \\
\hline \multicolumn{7}{|l|}{ 2) FRONTERA } \\
\hline a) Número & 406 & 398 & 420 & 480 & 551 & 543 \\
\hline b) Porcentaje & 90.6 & 89.8 & 91.9 & 88.9 & $88.9^{\circ}$ & 88.1 \\
\hline \multicolumn{7}{|c|}{ 3) RESTO DEL PAIS } \\
\hline a) Número & 42 & 45 & 37 & 60 & 69 & 73 \\
\hline b) Porcentaje & 9.4 & 10.2 & 8.1 & 11.1 & 11.1 & 11.9 \\
\hline
\end{tabular}

2. EMPLEO

1) TOTAL:
a) Número
74496
78433
$90704 \quad 111365 \quad 119546 \quad 131274$
b) Porcentaje
100.0100 .0
$\begin{array}{llll}100.0 & 100.0 & 100.0 & 100.0\end{array}$

2) FRONTERA
a) Número
$67532 \quad 70681$
$82387 \quad 100537$
$106576 \quad 116734$
b) Porcentaje
$90.6 \quad 90.1$
$90.8 \quad 90.3 \quad 89.1$
88.9

3) RESTO DEL PAIS
a) Número
$6949 \quad 7752$
$\begin{array}{lll}8317 & 10828 & 12970\end{array}$
14540
b) Porcentaje
$9.4 \quad 9.9$
9.2
$9.7 \quad 10.2$
11.1

3. VALOR AGREGADO

1) TOTAL:
a) Valor
4739
7118
10000
14543
$17729 \quad 23702$
b) Porcentaje
$100.0 \quad 100.0$
100.0
$100.0 \quad 100.0$
100.0

2) FRONTERA
a) Valor
$4196 \quad 5545$
7498
12005
20508
b) Porcentaje
88.5
$77.9 \quad 75.0$
82.5
$85.8 \quad 86.5$

3) RESTO DEL PAIS
a) Valor
543
$873 \quad 250$
2538
2522
3194
b) Porcentaje
11.5
22.1
25.0
17.5
14.2
13.5

a Millones de pesos a precios corrientes.

FUENTE: Calculado de: Secretaría de Patrimonio y Fomento Industrial. Mexico: empresas maquiladoras de exportación 1976-1981 (mimeo, 1982). 
y el empleo y valor agregado asociados con ellas durante el período 19761981. Los datos del cuadro muestan que durante ese período: un promedio de $89.7 \%$ de las maquiladoras de México fueron localizadas en la región fronteriza; ellas empleaban un promedio de alrededor de $90 \%$ del total de trabajadores de maquiladoras, y; representaban un promedio de alrededor de $83 \%$ del valor agregado por el total de maquiladoras en México.

Dentro de la región fronteriza, la distribución de las maquiladoras ha ido en favor de el lado oeste en términos del número de plantas, aunque no en términos de empleo. Por ejemplo, en 1981 alrededor del $40 \%$ de las plantas fronterizas fueron localizadas en el estado de Baja California, pero emplearôn sólo a cerca del $20 \%$ del número total de trabajadores empleados en las plantas fronterizas ese año. 25

\section{Turismo e información}

Muchos de los intercambios de turistas entre México y los Estados Unidos tienen lugar en su frontera común. Por ejemplo, en 1980, los gastos de turistas mexicanos en los Estados Unidos representaron el $21.1 \%$ de los gastos totales de turistas extranjeros en ese país, mientras que el $64.4 \%$ de los gastos de turistas mexicanos tuvieron lugar en la frontera de Estados Unidos con México. ${ }^{26}$

Con respecto a los turistas norteamericanos en México, en 1980 alrededor del $68 \%$ de ellos fueron residentes de los estados norteamericanos fronterizos con México y representaron más del $56 \%$ del valor total de los gastos de turistas norteamericanos en México. ${ }^{27}$

El intercambio de información es tal vez uno de los flujos más difíciles de medir directamente. Como una parte considerable del intercambio puede tener lugar a través del aire (radio y televisión) y por lo tanto sin otros elementos concomitantes, normalmente no puede ser registrado. La importancia de este flujo puede por lo tanto ser imputada sólo indirectamente a través de la evaluación de la infraestructura que existe en ambos lados de la frontera.

En 1978, los estados mexicanos fronterizos con los Estados Unidos contaban con alrededor de $24 \%$ del total de líneas telegráficas mexicanas;

25 En términos de empleo, las maquiladoras de C. Juárez incluyeron 37.8 del total de empleo de las plantas fronterizas en 1981. Véase: Secretaría de Patrimonio y Fomento Industrial, "México: emprcsas maquiladoras de exportación 1976-1981" (mimeo, 1982).

26 Véase: U.S. Department of Commerce, Statistical Abstract of the United States, 1981 p. 239.

27 Véase: Banco Nacional de México, S.A. Estadísticas básicas del turismo 1970-1980, México, 1981. 
$23 \%$ del total de líneas telefónicas; 19 por ciento del número de teléfonos, y $31 \%$ del número total de estaciones de aficionados del radio. ${ }^{28}$ También en 1978, los estados mexicanos fronterizos contaban con alrededor del $34.5 \%$ del total de estaciones de radio culturales y comerciales y $44.4 \%$ del número total de estaciones de televisión. ${ }^{29}$

Estas cifras se tornan más importantes en términos per capita; esto es, cuando las comparamos con la población del área fronteriza mexicana. En 1970, los estados mexicanos fronterizos con los Estados Unidos contaban con el $16.3 \%$ de la población total de México, y aunque para 1980 hay sólo datos preliminares, parece que dicha proporción ha decrecido recientemente. ${ }^{30}$

En el lado norteamericano de la frontera,, en 1979, los estados fronterizos representaban alrededor del $1.3 \%$ de las estaciones de radio nacionales y alrededor del $18 \%$ de las estaciones de televisión comercial de ese país. 31 .

Estas cifras también se tornan más importantes cuando se comparan con la población. En 1980, los estados norteamericanos fronterizos con México, representaban alrededor del $18.5 \%$ de la población total de esa nación. ${ }^{32}$

En general, la infraestructura de comunicaciones en la frontera sugiere que en el lado mexicano, la infraestructura per capita es más alta que en el lado americano. Sin embargo, la gran diferencia aparece cuando los números absolutos son comparados. Mencionando un ejemplo: mientras en los estados de la frontera mexicana había 12 estaciones de televisión en 1978, los estados de la frontera norteamericana tuvieron 136 estaciones comerciales de televisión. Por lo tanto, uno debe esperar una intensidad más fuerte de la información en la dirección Estados Unidos-México, por lo menos en estas dos consideraciones: radio y televisión.

La discusión anterior de las características generales de los procesos de interacción socioeconómica y cultural y de la distribución regional de los

\footnotetext{
28 Véase: Secretaría de Programación y Presupuesto, Manual de estadísticas básicas del sector comunicaciones y transportes (México, D.F.: SPP 1981), pp. 275-380.

29 Véase: Secretaría de Programación y Presupuesto, Como es México (México, D.F.:) SPP 1980), pp. 225-231.

30 Véase: Secretaría de Industria y Comercio, IX Censo General de Población y Vivienda, México. 1970; S.P.P., X Censo General de Población y Vivienda - Resultados preliminares a nivel nacional y por entidad federativa. México, 1981.

31 Véase: U.S. Department of Commerce. State and metropolitan area data book, Table C. Item 1697-1712.

32 Véase: U.S. Bureau of Census, 1980 Census of population supplementary report, P.C. 80-51-1
} 
principales flujos de intercambio entre México y los Estados Unidos sugiere que de hecho, la intensidad de los flujos es relativamente más fuerte en la frontera que en el resto de la nación, por dos razones: su tamaño y su duración.

Además, la receptividad de la sociedad norteamericana medida a prior por la diversidad étnica de su población. también se muestra más alta en la frontera que en el resto de la nación. Mientras que nacionalmente la gente de origen mexicano en 1980 representaba alrededor del $60 \%$ de la población total hispánica norteamericana (la cual a su vez es considerada como el $6.4 \%$ de la población total norteamericana), en los estados fronterizos representaban más del $80 \%$. Por otro lado, en ese año la población de origen hispánico de los estados fronterizos representaban el $57.8 \%$ de la población hispano-norteamericana total. (Véase el cuadro II).

CUADRO II. Población hispano-norteamericana y mexico-norteamericana por estados seleccionados. 1980 (miles de pesos).

\begin{tabular}{|c|c|c|c|c|}
\hline \multirow[t]{2}{*}{ ESTADO } & \multicolumn{2}{|c|}{ POBLACION HISPANICA } & \multicolumn{2}{|c|}{$\begin{array}{c}\text { POBLACION } \\
\text { MEXICO AMERICANA }\end{array}$} \\
\hline & $\begin{array}{c}\text { Número } \\
\text { (a) }\end{array}$ & $\begin{array}{l}\text { o/o } \\
\text { (b) }\end{array}$ & $\begin{array}{l}\text { Número } \\
\text { (c) }\end{array}$ & $\left(d^{o / o} \mathrm{c} / \mathrm{a}\right)$ \\
\hline Arizona & 440.9 & 100.0 & 396.0 & 89,8 \\
\hline California & $4,543.8$ & 100.0 & $3,600.0$ & 79.2 \\
\hline Nuevo Mexico & 476.1 & 100.0 & 233.0 & 48.9 \\
\hline Texas & $2,985.6$ & 100.0 & $2,800.0$ & 93.8 \\
\hline (1) FRONTERA & $8,446.4$ & 100.0 & $7,029.0$ & 83.2 \\
\hline (2) USA & $14,605.9$ & 100.0 & $8,763.5$ & 60.0 \\
\hline$(3)=(1) /(2)$ & 57.8 & & 80.2 & \\
\hline
\end{tabular}

FUENTE: Calculado de : U.S. Bureau of the Census, 1980 Census of Population, Supplementary Report, P.C. $80-51-1$

Con más altos grados de intensidad y receptividad del país anfitrión, los efectos del intercambio de flujos debieran ser más fuertes en la frontera que a nivel nacional en términos tanto culturales como económicos.

Reacción de la frontera a los intercambios de flujos

La cultura fue definida anteriormente como "ese todo complejo que incluye conocimiento, creencia, arte, moral, ley, costumbre y cualesquiera 
otras capacidades y hábitos adquiridos por el hombre como miembro de una sociedad" (Nota 3). Ha sido propuesto, sin embargo, que la herramienta singular más importante que define a la cultura es el idioma. ${ }^{33}$

Considerado como el instrumento más importante a la hora de transmitir conocimiento, el idioma participa en todos los procesos culturales, y llega a ser muy importante en las relaciones internacionales cuando los países involucrados no comparten la misma lengua.

Cualquier cambio en los patrones del uso del idioma o en cualquier otra manifestación o elemento cultural, significa un cambio cultural y/o adaptación. ${ }^{34}$ En el resto de esta sección, se analizará la reacción de la frontera a los flujos de intercambio, principalmente en dos consideraciones: culturalmente al través del patrón del uso del idioma y económicamente, al través del análisis del funcionamiento del sector externo de la economía fronteriza.

Idioma. Con respecto al idioma, se podría afirmar que la respuesta a la presencia de flujos de intercambio internacional ha sido más fuerte en los Estados Unidos que en México. Por ejemplo, después de que la Suprema Corte de Estados Unidos reglamentó en 1974 que "sin ayuda, los estudiantes que no hablan el lenguaje de la escuela, son efectivamente privados de una educación significativa.", 35 la sociedad americana empezó à reaccionar adoptando programas bilingües en sus escuelas primarias y secundarias. La importancia de esta reacción puede ser entendida de acuerdo al número de estudiantes de origen hispano inscritos en programas bilingües, los que, en 1976 ascendieron a 765,747 individuos en los Estados Unidos. Como es de esperarse, las inscripciones más grandes tuvieron lugar en los estados fronterizos, con casi el 63 del total. 36

La investigación llevada a cabo en 1982 en el Centro de Estudios Fronterizos del Norte de México (CEFNOMEX), sobre la relación entre el uso adecuado del idioma español y el nivel de identidad nacional en siete ciudades mexicanas incluidas tres poblaciones fronterizas, ha mostrado que no

33 Véase: Leslie White, op. cit.

34 El idioma es tal vez el elemento cultural al través del cual se puede detectar más directamente la influencia de una cultura externa. Sin embargo, es posible detectar tal influencia, al menos indirectamente, en otros elementos tales como el arte, el conocimiento, creencias, moral, leyes y el gusto. 35 Alan Pifer, Bilingual education and the Hispanic challenge (New York: Carnegie Corporation,
1979 )

36 Las inscripciones respectivas en los estados fronterizos fueron como sigue: Texas, 273, 880 estudiantes; California, 161,676, Nuevo México, 24,827, y Arizona, 20,172, Ibid. 
hay una diferencia significativa entre la frontera y el resto del país en términos de propiedad en el uso del lenguaje. ${ }^{37}$

Efectivamente, midiendo la impropiedad al través del uso de palabras y hábitos extranjeros (norteamericanos), las poblaciones fronterizas mostraron tanto el caso con el más alto nivel de uso de extranjerismos (Ciudad Juárez) como el caso del más bajo nivel (Matamoros), entre las siete ciudades estudiadas. 38 Midiendo la identidad nacional al través de una serie de indicadores de hábitos y tradiciones mexicanas, las poblaciones fronterizas mostraron también el caso de más alto nivel de identidad nacional (Ciudad Juárez). En este respecto la ciudad con el más bajo nivel de identidad nacional no fue una ciudad. fronteriza, sino la ciudad capital del país: México, D. F. 39

Estos resultados sugieren que los efectos de la relación cultural entre México y Estados Unidos no son significaticamente más fuertes (y mucho menos exclusivos) en la frontera mexicana, cuando el uso del idioma español es considerado.

El sector externo de la economía fronteriza. Una de las diferencias económicas importantes entre la frontera y el resto de los respectivos países, es su nivel de desarrollo. De hecho, el lado mexicano de la frontera es una de las áreas más desarrolladas de México considerando al producto regional bruto, ingreso per cápita, e índices generales de bienestar. En contraste, el lado de Estados Unidos en la frontera ha sido observado como una de las áreas de mayor depresión en los Estados Unidos.

Otra diferencia significativa entre las economías nacionales y las fronterizas, al menos para el caso de México recae en el comportamiento del sector externo. Por ejemplo, entre 1971 y 1981, la cuenta comercial de la balanza de pagos de México se ajustó más intensamente que por su contraparte fornteriza. ${ }^{40}$ Tal diferencia en el comportamiento alcanzó un extremo cuando, entre 1976 y 1977 (cuando tuvo lugar una devaluación del

\footnotetext{
37 Las siete ciudades estudiaças fueron: Tijuana, Ciudad Juárez y Matamoros en la Frontera; Acapulco, México D.F., Zacatecas y Uruapan en el resto del país. Véase: CEFNOMEX Uso del idioma español e identidad nacional, Tijuana, B.C. Centro de Estudios Fronterizos del Norte de México, 1982, (mimeo). La principal conclusión del estudio es que no hay una relación estadística significativa entre el uso del idioma español y la identidad nacional.

39 Ibid.

40 El análisis del sector externo de la economía fronteriza es posible porque las transacciones en general son registradas y publicadas en México como parte de la cuenta corriente de la balanza de pagos.
} 
peso) el valor de las exportaciones fronterizas descendió mientras que el de las exportaciones nacionales aumentó. ${ }^{41}$

Otras reacciones económicas y culturales. La interdependencia y las convergencias en la frontera pueden ser detectadas indirectamente también mediante otros indicadores menos formales. El grado del uso de dólares como moneda legal a lo largo del lado mexicano de la frontera y la aceptación de pesos mexicanos como pago en los negocios americanos; o el siempre creciente número de restaurantes norteamericanos que ofrecen comida mexicana; o el alto porcentaje de universidades norteamericanas en los estados fronterizos que ofrecen estudios mexico-norteamericanos, no son sino pocos ejemplos de los cambios culturales y/o las adaptaciones económicas que se están dando en la frontera.

\section{RESUMEN Y CONCLUSIONES}

Como se mencionó en la introducción, este trabajo trata de la frontera México-Estados Unidos y de la medida en la que lo que sucede en esta área está relacionado con el proceso de latinoamericanización de los Estados Unidos.

Para explorar este proceso de latinoamericanización, se desarrolló un modelo para calificar los efectos del intercambio internacional que tiene lugar entre los dos países. En tal modelo, el cambio cultural y la adaptación económica, son de función de la intensidad de los flujos de intercambio, y de la receptividad o disponibilidad/habilidad al cambio del país anfitrión:

La evidencia empírica presentada aquí sugiere que porque nacionalmente el principal flujo México-Estados Unidos es la migración de trabajadores, mientras el principal flujo de Estados Unidos-México es el capital físico y la tecnología, el impacto cultural es más fuerte en Estados Unidos que en México. La evidencia también sugiere que considerando la migración, el capital físico, el turismo y la información, los impactos culturales y socioeconómicos del intercambio internacional son más intensos en la región fronteriza que en el resto de la nación.

Estas consideraciones llevan a la conclusión de que la frontera MéxicoEstados Unidos es el lugar donde las convergencias cultural y socioeconómica de los dos países es más intensa y donde, por la naturaleza y la intensidad de las interacciones, el proceso de latinoamericanización de los Estados Unidos es más intenso y aparente que el de la norteamericanización de Latinoamérica.

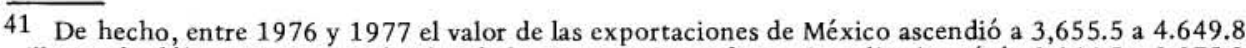
millones de dólares, mientras el valor de las exportaciones fronterizas disminuyó de 2.266 .5 a 2.075.9 millones de dólares. Véase: Banco de México, Indicadores Económicos, varios volúmenes. 
peso) el valor de las exportaciones fronterizas descendió mientras que el de las exportaciones nacionales aumentó. ${ }^{41}$

Otras reacciones económicas y culturales. La interdependencia y las convergencias en la frontera pueden ser detectadas indirectamente también mediante otros indicadores menos formales. El grado del uso de dólares como moneda legal a lo largo del lado mexicano de la frontera y la aceptación de pesos mexicanos como pago en los negocios americanos; o el siempre creciente número de restaurantes norteamericanos que ofrecen comida mexicana; o el alto porcentaje de universidades norteamericanas en los estados fronterizos que ofrecen estudios mexico-norteamericanos, no son sino pocos ejemplos de los cambios culturales y/o las adaptaciones económicas que se están dando en la frontera.

\section{RESUMEN Y CONCLUSIONES}

Como se mencionó en la introducción, este trabajo trata de la frontera México-Estados Unidos y de la medida en la que lo que sucede en esta área está relacionado con el proceso de latinoamericanización de los Estados Unidos.

Para explorar este proceso de latinoamericanización, se desarrolló un modelo para calificar los efectos del intercambio internacional que tiene lugar entre los dos países. En tal modelo, el cambio cultural y la adaptación económica, son de función de la intensidad de los flujos de intercambio, y de la receptividad o disponibilidad/habilidad al cambio del país anfitrión:

La evidencia empírica presentada aquí sugiere que porque nacionalmente el principal flujo México-Estados Unidos es la migración de trabajadores, mientras el principal flujo de Estados Unidos-México es el capital físico y la tecnología, el impacto cultural es más fuerte en Estados Unidos que en México. La evidencia también sugiere que considerando la migración, el capital físico, el turismo y la información, los impactos culturales y socioeconómicos del intercambio internacional son más intensos en la región fronteriza que en el resto de la nación.

Estas consideraciones llevan a la conclusión de que la frontera MéxicoEstados Unidos es el lugar donde las convergencias cultural y socioeconómica de los dos países es más intensa y donde, por la naturaleza y la intensidad de las interacciones, el proceso de latinoamericanización de los Estados Unidos es más intenso y aparente que el de la norteamericanización de Latinoamérica.

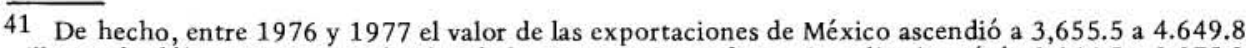
millones de dólares, mientras el valor de las exportaciones fronterizas disminuyó de 2.266 .5 a 2.075.9 millones de dólares. Véase: Banco de México, Indicadores Económicos, varios volúmenes. 